\title{
Is there a genetic basis to the different morphological subtypes of bicuspid aortic valve?
}

\author{
Talha Niaz ${ }^{1}$, Donald J. Hagler ${ }^{1,2}$ \\ ${ }^{1}$ Department of Pediatrics, Division of Pediatric Cardiology, ${ }^{2}$ Department of Medicine, Department of Cardiovascular Diseases, Mayo Clinic, \\ Rochester, MN, USA \\ Correspondence to: Donald J. Hagler, MD. Divisions of Pediatric Cardiology and Cardiovascular Diseases, Mayo Clinic College of Medicine, 200 First \\ Street SW, Rochester, MN 55905, USA. Email: hagler.donald@mayo.edu. \\ Provenance: This is an invited Editorial commissioned by Section Editor Ran Mo, MD (Department of Cardiothoracic Surgery, Nanjing Drum Tower \\ Hospital, the Affiliated Hospital of Nanjing University Medical School, Nanjing, China). \\ Comment on: Gharibeh L, Komati H, Bossé Y, et al. GATA6 regulates aortic valve remodeling, and its haploinsufficiency leads to right-left type \\ bicuspid aortic valve. Circulation 2018;138:1025-38.
}

Submitted Oct 25, 2018. Accepted for publication Dec 10, 2018.

doi: 10.21037/atm.2018.12.21

View this article at: http://dx.doi.org/10.21037/atm.2018.12.21

Bicuspid aortic valve (BAV) is one of the most common congenital heart defects leading to a significant global financial health burden as a result of its complications (1). $\mathrm{BAV}$ can lead to premature aortic valve disease in the form of aortic valve stenosis or regurgitation requiring multitude of interventions on aortic valve, including transcatheter interventions or surgical valve replacement. BAV disease is a continuum of disease process that not only affects the aortic valve but also extends to the thoracic aorta leading to dilatation with risk of aortic aneurysm and dissection. Even though the impacts of BAV are far and wide spread and despite decades of research and investigation, we still lack a clear understanding of the genetic and molecular pathophysiology of BAV (2).

The study by Gharibeh et al. describes the role of GATA6 in regulation of aortic valve remodeling as a novel cellular and molecular mechanism underlying BAV (3). This is a very well-orchestrated study in which authors analyzed GATA6 gene variants in $452 \mathrm{BAV}$ cases and 1,849 controls from Framingham GWAS study. Authors also delved into the expression of GATA6; GATA6 haploinsufficiency leads to reduced levels of tissue inhibitor of metalloproteinase 1 (TIMP1), fibrillin 1 (FBN1), and Versican (VCAN), which play an important role in the integrity of extracellular matrix and could be a potential pathophysiological mechanism of abnormal valvulogenesis in BAV. Moreover, GATA6 haploinsufficiency also leads to an abnormal apoptosis and thus abnormal valve remodeling.

The GATA family of zinc-finger transcription factors plays an essential role in cardiac embryogenesis especially GATA4, GATA5 and GATA6. They are expressed in the endocardial cushions, outflow tract and cardiac neural crest cells which are important in development of aortic valve. Variants with GATA5 and GATA6 have been observed in patients with BAV and indirectly provide a link to BAV formation (4). Interestingly all the BAVs in this study by Gharibeh et al. were RL in morphology, which leads to a strong association of GATA6 mutation with RL cusp fusion. In prior studies, Laforest et al. have demonstrated the association of GATA5 with RN cusp fusion morphology; where they generated GATA5 null mice models that showed a partial penetrance of BAV and all these cases of BAV demonstrated RN cusp fusion (5). This highly supports the idea of distinct genetic differences among the morphological subtypes of BAV possibly leading to variations in the developmental pathway of aortic valve formation. In order to comprehend these differences, we need to understand the embryogenesis of BAV and its morphological subtypes. The right and left cusps of aortic valve arise from the conotruncal cushions of outflow tract, while the non-coronary (LN) cusp arises from the rightposterior and left-anterior intercalated cushions of the outflow tract $(4,6)$. Some recently published studies in mice and Syrian hamster also suggested that BAV with RN cusp 
fusion results from defective development of the outflow tract cushions, while BAV with RL cusp fusion possibly results from abnormal septation of proximal outflow tract region $(7,8)$. Therefore, morphological phenotypes of BAV may have distinct embryological origins.

Authors in this study also evaluated cardiac neural crest cell dysregulation which was normally regulated in GATA6 haploinsufficient mice. Although this does not seem to be a mechanism in BAV with RL morphology, it could still be a potential mechanism of abnormal valvulogenesis in BAV with RN morphology. Recently Odelin et al. reported the loss of Krox20 function, which has been implicated in cardiac neural crest cells regulation, resulting in partially penetrant $\mathrm{BAV}$ in $\mathrm{Krox} 20^{-/-}$mice. Interestingly, the phenotype of $\mathrm{BAV}$ in $\mathrm{Krox} 20^{-/-}$mice was either $\mathrm{RN}$ or left non-coronary (LN) cusp fusion. $\mathrm{RN}$ cusp fusion is also reported in patients with Down syndrome and atrioventricular septal defects, which is mainly caused by dysregulation of neural crest cells and may provide an indirect association (6). Considering the association of GATA5 with RN cusp fusion, it will be valuable to examine cardiac neural crest cell dysregulation in GATA5 haploinsufficient or null mice which could be another potential pathophysiological mechanism of abnormal valve formation in BAV with $\mathrm{RN}$ morphology $(5,9)$.

Authors further validated the study by looking at the variants within the GATA6 gene in human subjects which although did not reach the locus wide significance but showed a decreased expression of GATA6 in valves and aorta of individuals with BAV in comparison to tricuspid aortic valves. We congratulate the authors on excellent study and development of an animal model which opens up the doors for further investigations, not only related to aortic valve but also aortopathy.

\section{Acknowledgements}

None.

\section{Footnote}

Conflicts of Interest: The authors have no conflicts of interest to declare.

\section{References}

1. Badheka AO, Singh V, Patel NJ, et al. Trends of Hospitalizations in the United States from 2000 to 2012 of Patients $>60$ Years With Aortic Valve Disease. Am J Cardiol 2015;116:132-41.

2. Michelena HI, Prakash SK, Della Corte A, et al. Bicuspid aortic valve: identifying knowledge gaps and rising to the challenge from the International Bicuspid Aortic Valve Consortium (BAVCon). Circulation 2014;129:2691-704.

3. Gharibeh L, Komati H, Bosse Y, et al. GATA6 Regulates Aortic Valve Remodeling and its Haploinsufficiency Leads to RL-Type Bicuspid Aortic Valve. Circulation 2018;138:1025-38.

4. Martin PS, Kloesel B, Norris RA, et al. Embryonic Development of the Bicuspid Aortic Valve. J Cardiovasc Dev Dis 2015;2:248-72.

5. Laforest B, Andelfinger G, Nemer M. Loss of Gata5 in mice leads to bicuspid aortic valve. J Clin Invest 2011;121:2876-87.

6. Niaz T, Poterucha JT, Olson TM, et al. Characteristic Morphologies of the Bicuspid Aortic Valve in Patients with Genetic Syndromes. J Am Soc Echocardiogr 2018;31:194-200.

7. Fernández B, Duran AC, Fernandez-Gallego T, et al. Bicuspid Aortic Valves With Different Spatial Orientations of the Leaflets Are Distinct Etiological Entities. J Am Coll Cardiol 2009;54:2312-8.

8. Odelin G, Faure E, Coulpier F, et al. Krox20 defines a subpopulation of cardiac neural crest cells contributing to arterial valves and bicuspid aortic valve. Development 2018;145.

9. Padang R, Bagnall RD, Richmond DR, et al. Rare nonsynonymous variations in the transcriptional activation domains of GATA5 in bicuspid aortic valve disease. J Mol Cell Cardiol 2012;53:277-81.

Cite this article as: Niaz T, Hagler DJ. Is there a genetic basis to the different morphological subtypes of bicuspid aortic valve? Ann Transl Med 2018;6(Suppl 2):S117. doi: 10.21037/ atm.2018.12.21 